\title{
Cytomegalic Infection in an Immunocompetent Patient: A Case With Multiple Organ Dysfunction
}

\author{
Joana Urbano ${ }^{\mathrm{a}, \mathrm{b}, \mathrm{e}}$, Sofia Moreira-Silva ${ }^{\mathrm{a}, \mathrm{b}}$, Fernando Frioes ${ }^{\mathrm{b}, \mathrm{c}}$, Jorge Almeida ${ }^{\mathrm{b}, \mathrm{c}}$, \\ Candida Abreu ${ }^{\mathrm{b}, \mathrm{d}}$, Joana Pimenta ${ }^{\mathrm{a}, \mathrm{b}}$
}

\begin{abstract}
Cytomegalic infection of the immunocompetent adult is usually asymptomatic or expressed by a mononucleosic syndrome. We present a case of a young adult with rare and severe primary cytomegalic infection and multisystemic involvement, namely hematological, hepatic, gastrointestinal and respiratory, including pneumonia with severe pulmonary hypertension, to the best of our knowledge, not previously described. Also of note is the fact that these dysfunctions were treated successfully with ganciclovir, although a formal indication to antivirals on cytomegalic infection of the immunocompetent patients is not established yet.
\end{abstract}

Keywords: Immunocompetent; Adult; Cytomegalovirus; Ganciclovir

\section{Introduction}

Immunocompetent cytomegalic infection is characteristically asymptomatic or expressed by a mononucleosic syndrome [1]. Severe disease or multiple organ involvement is rare, so antiviral drugs are not routinely recommended $[2,3]$. We report a case of a patient that illustrates two uncommon aspects of cytomegalic infection on immunocompetent individuals: severe multiple organ involvement, and the utility of antiviral drugs in that setting. Also of note is that the patient had diffuse pulmonary involvement causing acute cor pulmonale, as far as we know, just previously described associated with pulmonary embolism and not due to parenquimal attainment.

\footnotetext{
Manuscript accepted for publication August 13, 2015

anternal Medicine Department, Centro Hospitalar Sao Joao, Porto, Portugal bUniversity of Porto Medical School, Porto, Portugal

'Intermediate Care Unit of Medicine Department, Centro Hospitalar Sao Joao, Porto, Portugal

dinfectious Diseases Department, Centro Hospitalar Sao Joao, Porto, Portugal ${ }^{e}$ Corresponding Author: Joana Urbano, Internal Medicine Department, Centro Hospitalar Sao Joao, Alameda Professor Hernani Monteiro, 4200-319 Porto, Portugal. Email: joanaurb@gmail.com
}

doi: http://dx.doi.org/10.14740/jmc2265w

\section{Case Report}

A 34-year-old man presented to the emergency department of our institution with a 1-week history of fever, myalgia, fatigue, loss of appetite, nausea, non-bloody diarrhea and intense right temporal headache. He had a history of light smoking habits ( 0.5 packs per year) and epilepsy, medicated with carbamazepine since childhood. On admission, his physical examination revealed fever, tachycardia, tachypnea, paleness and dehydration. On abdominal exam, he had both enlarged liver and spleen. Laboratory tests showed moderate lymphocytosis and normal $\mathrm{C}$ reactive protein. He was discharged with the diagnosis of acute non-bacterial enteritis and symptomatic medication was prescribed.

One week later, besides worsening diarrhea, he developed sore throat and serious retroocular headache, with intense photophobia. His wife reported an episode of delirium, with acute confusion and incoherent speech the night before. He was brought again to the emergency department, where he presented with a slightly ataxic gate with a left side deviation, fever, and swollen inflamed tonsils. The remainder neurological exam, as well as abdominal and chest examination were normal. Laboratory tests showed increased lymphocytosis with atypical lymphocytes on peripheral blood smear, as well as moderate normocytic anemia and thrombocytopenia of $115,000 / \mathrm{mm}^{3}$. C reactive protein was $61.3 \mathrm{mg} / \mathrm{L}$ (normal $<3 \mathrm{mg} / \mathrm{L}$ ) and transaminases levels increased to almost two times the upper reference limit, with serum albumin of $32 \mathrm{~g} / \mathrm{L}$. Bilirubin and prothrombin time were within the normal range. Cerebral CT scan was nor$\mathrm{mal}$, and no signs of infection were found in cerebrospinal fluid analysis. He was then admitted to internal medicine ward with suspected mononucleosic infection.

To exclude other etiologies of diarrhea, bacteriologic and parasitological fecal exams, fecal leucocytes testing and Widal reaction were performed, which were negative. He had a positive fecal occult blood test. Serological tests for Epstein-Barr virus, Coxiella burnetii, varicella zoster, herpes simplex virus (HSV), Toxoplasma gondii, and A, B and C hepatitis were negative. Cytomegalovirus (CMV) serology showed positive $\mathrm{IgM}$ and $\mathrm{IgG}$ titers, and blood polymerase chain reaction revealed $5.5 \times 10^{4}$ copies $/ \mathrm{mL}$ of CMV DNA. Both primary and acquired immunodeficiencies were excluded by a negative human immunodeficiency virus test and normal concentration of immunoglobulin subtypes (IgG, IgA and IgM). Because of 
persisting neurological symptoms, with recurrent episodes of confusion and somnolence, lumbar puncture was repeated and a cerebral MRI was performed, both without alterations. No CMV, Listeria, enterovirus or HSV were detected in the cerebrospinal fluid. Electroencephalogram showed slow and irregular traces reflecting global cerebral dysfunction, without epileptic discharges. The patient developed worsening hematological dysfunction: minimum platelet count was 77,000/ $\mathrm{mm}^{3}$ and hemoglobin dropped to $9.9 \mathrm{~g} / \mathrm{dL}$. Search for schizocytes was negative and lactic dehydrogenase level was within the normal range. Patient reported blurred vision and the ophthalmologic exam showed a little retinal hemorrhagic infiltrate and no signs of cytomegalic retinitis.

A few days later, symptoms of breathlessness and dry cough started and severe hypoxia was documented (ratio $\mathrm{PaO}_{2} /$ $\mathrm{FiO}_{2}$ 152). A thoracic CT scan identified enlarged mediastinal and hilar lymph nodes, as well as pulmonary micronodules, ground-glass parenchymal areas and small pleural and pericardial effusions, and showed no signs of pulmonary embolism. Transthoracic echocardiogram showed severe pulmonary hypertension (pulmonary artery systolic pressure of $70 \mathrm{~mm} \mathrm{Hg}$ ) and signs of acute cor pulmonale. At this time, patient was transferred to the intermediate care unit, where he began intravenous ganciclovir, at the dose of $5 \mathrm{mg} / \mathrm{kg}$. Antiviral therapy was maintained for 21 days, with gradual improvement of the patient condition, progressive correction of respiratory failure, and neurological and hematological dysfunction.

The patient was discharged 26 days after admission, with complete resolution of respiratory insufficiency. He was reevaluated periodically on the ambulatory clinic, repeating studies to exclude congenital or acquired immune deficiencies, including autoimmune diseases and malignancies. Pulmonary hypertension showed slow but complete resolution, as well as neurological deficits, hepatic and splenic enlargement, platelet count and hemoglobin. Resolution of constitutional symptoms was slower and occurred only after 18 months. IgG titers increased to a maximum of 1,207 AU/mL 11 months after the initial infection, with documented seroconversion after that.

\section{Discussion}

This case documents an unusual presentation of CMV infection in an immunocompetent adult patient, with severe multisystemic involvement. We reviewed previous reports of severe CMV infection in immunocompetent adults, usually documenting single organ or system dysfunction [2-4]. Among these reports, the gastrointestinal [5-7] and respiratory tract [8], as well as central nervous system [3], were the most frequent sites of severe CMV infection. Other manifestations included hematological disorders (hemolytic anemia and thrombocytopenia) [9, 10], thrombosis of the venous or arterial vasculature [11] and ocular involvement [12]. In contrast to those reports, our patient presented with an unusual clinical scenario of important multisystemic involvement, namely hematological, neurological, enteric and respiratory. Besides he developed severe pulmonary hypertension, with acute cor pulmonale, to the best of our knowledge, not previously described due to parenquimal disease. Previous reports of pulmonary hypertension were asso- ciated with acute pulmonary embolism, which was not the case.

This case also stresses the efficacy of antiviral therapy in severe CMV infection in an immunocompetent patient, as the patient dysfunctions responded successfully to ganciclovir. There are some previous reports on the use of antiviral treatment for the most severe manifestations of cytomegalic infection, documenting good patient outcome [4, 7]. However, a formal indication to use ganciclovir in adult immunocompetent cytomegalic infection is still not established [2].

\section{Grant Support}

Not applicable.

\section{Competing Interests}

The authors declare that they do not have any competing interests.

\section{References}

1. Wreghitt TG, Teare EL, Sule O, Devi R, Rice P. Cytomegalovirus infection in immunocompetent patients. Clin Infect Dis. 2003;37(12):1603-1606.

2. Lancini D, Faddy HM, Flower R, Hogan C. Cytomegalovirus disease in immunocompetent adults. Med J Aust. 2014;201(10):578-580.

3. Rafailidis PI, Mourtzoukou EG, Varbobitis IC, Falagas ME. Severe cytomegalovirus infection in apparently immunocompetent patients: a systematic review. Virol J. 2008;5:47.

4. Orasch C, Conen A. Severe primary cytomegalovirus infection in the immunocompetent adult patient: a case series. Scand J Infect Dis. 2012;44(12):987-991.

5. Karakozis S, Gongora E, Caceres M, Brun E, Cook JW. Life-threatening cytomegalovirus colitis in the immunocompetent patient: report of a case and review of the literature. Dis Colon Rectum. 2001;44(11):1716-1720.

6. Karigane D, Takaya S, Seki Y, Mastumoto Y, Onose A, Kosakai A, Sugaya N, et al. Cytomegalovirus enteritis in immunocompetent subjects: a case report and review of the literature. J Infect Chemother. 2014;20(5):325-329.

7. Nazir S, Eledrisi M. Cytomegalovirus Infection in an Immunocompetent Host Presenting With Partial Bowel Obstruction. J Med Cases. 2015;6(4):153-155.

8. Grilli E, Galati V, Bordi L, Taglietti F, Petrosillo N. Cytomegalovirus pneumonia in immunocompetent host: case report and literature review. J Clin Virol. 2012;55(4):356359.

9. Nomura K, Matsumoto Y, Kotoura Y, Shimizu D, Kamitsuji Y, Horiike S, Tamiwaki M. Thrombocytopenia due to cytomegalovirus infection in an immunocompetent adult. Hematology. 2005;10(5):405-406.

10. Miyahara M, Shimamoto Y, Yamada H, Shibata K, Matsuzaki M, Ono K. Cytomegalovirus-associated myelod- 
ysplasia and thrombocytopenia in an immunocompetent adult. Ann Hematol. 1997;74(2):99-101.

11. Ofotokun I, Carlson C, Gitlin SD, Elta G, Singleton TP, Markovitz DM. Acute cytomegalovirus infection complicated by vascular thrombosis: a case report. Clin Infect
Dis. 2001;32(6):983-986.

12. Radwan A, Metzinger JL, Hinkle DM, Foster CS. Cytomegalovirus retinitis in immunocompetent patients: case reports and literature review. Ocul Immunol Inflamm. 2013;21(4):324-328. 